\title{
Ambulatory Blood Pressure Control Pattern in Hypertensive Patients at Tikur Anbesa Specialized Hospital: A Cross Sectional Study
}

\section{MULUALEM ALEMAYEHU ( $\sim$ mulualemalemayehuf@gmail.com )}

Addis Ababa University

\section{Sintayehu Abebe}

Addis Ababa University

Dejuma Yadeta

Addis Ababa University

Bekele Alemayehu

Addis Ababa University

\section{Research Article}

Keywords: cardiovascular problem, ABPM control, BP control, Hospitals outpatient departments

Posted Date: September 20th, 2021

DOI: https://doi.org/10.21203/rs.3.rs-763121/v1

License: (c) (i) This work is licensed under a Creative Commons Attribution 4.0 International License. Read Full License 


\section{Abstract}

Background: Hypertension is the most common cardiovascular problem globally with a particularly increasing burden in developing countries like Ethiopia. Ambulatory blood pressure (ABPM) is superior to office blood pressure (OBP) measurement for diagnosing, prognosticating and following treatment efficacy for hypertension. There is no available data on ABPM control pattern in Ethiopians. This study will determine the ABPM control patterns in Ethiopian hypertensive patients on treatment.

Material and Methods: This was a cross sectional study in hypertensive patients at Tikur Anbessa Specialized Hospitals outpatient departments carried out during January to May 2021. ABPM values of 244 consecutively sampled patients were analyzed. All patients had their BP monitored over $24 \mathrm{~h}$ with a Tonoport V (GE CS V6 71), and the data was interpreted using GE CardiosoftTM ABPM software in accordance with European Society of hypertension guidelines. Ethical clearance was given by Addis Ababa University Institutional Review Board and the study was conducted in compliance to standard ethical guidelines.

Results: The study involved 244 adult hypertensive patients; mean age of the patients was $59.4 y e a r s$ and, $54 \%$ were females. $58.6 \%$ of patients had controlled OBP, while only $45.1 \%$ had controlled ABPM. The mean OBP was 137 (19)/81 (10) mmHg and mean 24-hr ABP was 137 (16)/81 (10) mmHg; mean daytime $B P$ was 136/79 $\pm 17 / 11 \mathrm{mmHg}$; mean night-time BP, 138/84 \pm 16/11 mmHg. Mean ABPM values were not significantly different between men and women. Comparison of ABPM values with OBP revealed high prevalence of the white coat effect (32\%) and masked uncontrolled hypertension (46\%). Presence of comorbidities particularly diabetes predicted poor ABPM control.

Conclusion: More than half of patients had uncontrolled BP as per ABPM criteria and significant discrepancy exists between ABPM and OBP in assessing adequacy of BP control. Guiding management decisions using $A B P M$ can improve BP control rates.

\section{Background}

Hypertension defined as a systolic and/or diastolic blood pressure $\geq 140 / 90 \mathrm{mmHg}$, is the most common cardiovascular disease globally. It is a major cardiovascular risk factor and a leading risk factor for global deaths. Raised BP remains the leading cause of death globally, accounting for 10.4 million deaths per year (Global Burden of Disease, 2018). When reviewing global figures, an estimated 1.39 billion people had hypertension in 2010. The highest prevalence of raised blood pressure was seen in the African Region (27\%).(1)

In Ethiopia, $16 \%$ of the adult population has raised blood pressure with similar prevalence seen in males and females. The overwhelming majority of hypertensive Ethiopians do not take any blood pressure lowering medication. Of those patients on treatment only $53 \%$ had controlled hypertension.(2) 
It has been well recognized that office BP measurements have significant limitation in diagnosing and prognosticating hypertensive patients. Among the more precise methods of BP measurement, 24-hour (ambulatory) BP measurement has gained a wide acceptance. Ambulatory blood pressure monitoring generates much more information than the single (typically daytime) 'snapshot in time' reading yielded by clinic measurement by providing a profile of blood pressure behavior over a 24-hour period and blood pressure response to antihypertensive therapy in this period. It also allows more fine-tuned assessments during specific windows of this time cycle i.e. day vs. nighttime control. Night time blood pressure, nighttime dipping pattern and morning surge are important measures of this circadian variation and have significant prognostic implications.(3)

Achieving adequate blood pressure target in hypertensive patients is a difficult task even in the best of setups whereby half of the treated patients have uncontrolled BP(4). The prevalence of uncontrolled hypertension is even higher in developing countries where close to two thirds of patients on treatment fail to achieve targets and this contributes to worsened outcomes including premature death according to a recent review. Even among African countries, eastern African countries have the lowest proportion of treated patients and control rate.(5) A recent multi-center cross sectional study in Ethiopia showed that the rate of uncontrolled hypertension reaches up to $67 \%$ in patients on treatment for hypertension. (6)

A white-coat effect can also occur in patients with treated hypertension, and is particularly relevant to the evaluation of patients with possible resistant hypertension. Nearly $30-40 \%$ of patients with uncontrolled office BP have controlled blood pressure on ambulatory blood pressure monitoring.(7) In a larger Spanish study of over 8,200 patients with resistant hypertension, $38 \%$ were attributed to white-coat effect based on ambulatory blood pressure monitoring. Profile. (8)

On the other end of the spectrum, a masking phenomena occurs in hypertensives on treatment who apparently have normal office BP but ABPM values reveal poor control and is termed Masked Uncontrolled Hypertension (MUCH). Prevalence rates of up to $30 \%$ are reported in multiple ABPM studies. (9)

Discrepant rates of controlled BP have been reported with ABPM vs conventional BP in some populations.(10)(11)

\section{Methods}

The Ethics Committees of Addis Ababa University, Tikur Anbesa Specialized Hospital, Ethiopia, approved the study protocol and consent form (Dr. Adamu Adissie, Addis Ababa University Institutional Review Board chairperson). The participants gave informed written consent to the study. The study was conducted in accordance with the standard international and national scientific and ethical guidelines.

It is a cross sectional study, with data collected between January 2021 to May 2021. The study population consists of adult hypertensives 18 years and above at renal and cardiac clinics of TASH, who gave consent to be part of the study. Participants were consecutively recruited, demographic data, 
medical history, imaging, laboratory, and treatment history were collected from the electronic medical record.

OBP was measured in both arms with an oscillometric BP device, while the patient was sitting. The arm with the higher reading was taken as the patients BP after an average of three readings. Systolic and diastolic (Phase V) BP were determined to the nearest $2 \mathrm{mmHg}$ in accordance with European Society of Hypertension recommendation. Ambulatory BP was measured with the Tonoport V monitor (GE CS V67) for a minimum of $24 \mathrm{~h}$, using a similar sized cuff as was used in the OBP measurement. The ABP monitor measured BP at 30-min intervals for 24 hours; from $0600 \mathrm{~h}$ to $2200 \mathrm{~h}$ (representing daytime) and from 2200 to $0600 \mathrm{~h}$ (representing night-time). Participants kept a diary card for the duration of the record to note bedtime and waking time to define day and night and to check the transition time. ABPM data were interpreted in accordance with ESC Hypertension guidelines.

\section{STATISTICAL ANALYSIS}

ABPM data processing was performed using the GE Cardiosoft ${ }^{T M}$ Ambulatory Blood Pressure software (PAR Medizintechnik GmbH Sachsendammb D-10829, Berlin, Germany). Data was collected using the electronic data collection system (ODK Collect). This data was converted into excel format via the ODK briefcase software. Management of data and Statistical analysis was performed using the SPSS software package for Windows version 20 (SPSS Inc., Chicago, IL).

Baseline demographic data was analyzed using descriptive statistics. Correlation between predictor variables like age and office BP with the outcome variable (ABPM values) was analyzed by pearsonspearman coefficients. Factor analysis was used to identify representative laboratory variables in each category.

Comparison between groups (by sex, type of medication, type of comorbidity and OBP control status and number of HTN drugs) for ABPM values was done using the parametric variance tests (Two-tailed Student $T$ test and 1 way ANOVA). The non - parametric tests (chi square and kappa measure of agreement) were used to compare patients based on sex, type of anti-hypertensive medications ,type of comorbidity and OBP control status with $24 \mathrm{hr}$ ABPM ,mean day and mean night BP control status. Proportions were reported as percentages and compared between groups with Chi-square. A p-value less than 0.05 is considered statistically significant.

\section{Results}

The study involved 244 adult hypertensive patients having followed at TASH cardiac or renal clinics. The mean age of the cohort was 59.4 years, and $54 \%$ of subjects were female. Significant proportion of patient had comorbidities with $29 \%$ of patients having diabetes, $56 \%$ dyslipidemic or on statin therapy, 
and $16 \%$ having IHD. Majority of patients (65\%) were taking more than 1 anti-hypertensive drug (Table 1).

Table 1. Baseline demographic data

\begin{tabular}{|c|c|c|}
\hline PARAMETER & Number of Patients & percentage of Patients \\
\hline total patients & 244 & \\
\hline Mean Age (years) & 59.4 years & \\
\hline Females & 132 & $54 \%$ \\
\hline \multicolumn{3}{|c|}{ Type of anti-hypertensive } \\
\hline CCB & 150 & 61.5 \\
\hline ACEI & 147 & 60.2 \\
\hline ARB & 19 & 7.8 \\
\hline HCT & 63 & 25.8 \\
\hline BB & 75 & 30.7 \\
\hline Other anti-HTN & 21 & 8.6 \\
\hline \multicolumn{3}{|c|}{ Number of anti-hypertensives } \\
\hline 1 & 83 & 34 \\
\hline 2 & 91 & 37 \\
\hline 3 & 55 & 23 \\
\hline 4 & 13 & 5 \\
\hline 5 & 1 & .4 \\
\hline COMORBIDITY & NUMBER & PERCENT \\
\hline DIABETES & 70 & 28.7 \\
\hline DYSLIPIDEMIA & 136 & 55.7 \\
\hline STROKE & 5 & 2 \\
\hline IHD & 38 & 15.6 \\
\hline
\end{tabular}

Baseline laboratory and imaging data from echocardiography, doppler ultrasound of peripheral vessels and electrocardiogram revealed the extent of comorbidities and hypertension mediated target organ damage (Table 2).

Table 2. Baseline laboratory and imaging_parameters 


\begin{tabular}{|llllll|}
\hline Parameter & N & Minimum & Maximum & Mean & Std. Deviation \\
\hline creat & 114 & 0.6 & 11 & .97 & 1.132 \\
\hline fbs & 165 & 60 & 413 & 123.39 & 44.754 \\
\hline hga1c & 56 & 4 & 138 & 9.20 & 17.594 \\
\hline tc & 164 & 100 & 321 & 170.51 & 48.632 \\
\hline Idl & 155 & 26 & 238 & 120.66 & 40.721 \\
\hline hdl & 168 & 18 & 96 & 46.79 & 11.545 \\
\hline tg & 167 & 42 & 469 & 133.98 & 68.772 \\
\hline sv1_rv5_6 & 5 & 1 & 4 & 2.80 & 1.095 \\
\hline ravl & 5 & 1 & 3 & 1.40 & .894 \\
\hline ivs_thickness & 124 & 6 & 16 & 11.32 & 2.207 \\
\hline
\end{tabular}

BP control was suboptimal as shown from the mean of both OBP 137 (19)/81 (10) mmHg and 24-h ABP 137 (16)/81 (10) $\mathrm{mmHg}$. Rates of controlled BP were $58.6 \%$ for OBP, $45.1 \%$ for mean 24-h ABPM and only $21.7 \%$ for mean night time BP (Table 3 ).

Table 3. Results of ABPM and OBPM 


\begin{tabular}{|llllll|}
\hline & sample & $\min$ & $\max$ & Mean & SD \\
\hline Office SBP & 244 & 90 & 200 & 137.52 & 19.058 \\
\hline Office DBP & 244 & 60 & 120 & 81.30 & 10.929 \\
\hline Mean 24hr DBP & 244 & 60 & 120 & 80.95 & 10.371 \\
\hline mean_day_SBP & 244 & 102 & 198 & 135.45 & 17.576 \\
\hline mean_day_DBP & 244 & 60 & 120 & 79.30 & 11.075 \\
\hline mean_night_SBP & 244 & 100 & 198 & 138.41 & 16.247 \\
\hline mean_night_DBP & 244 & 62 & 125 & 84.09 & 11.546 \\
\hline maximum 24hr SBP & 244 & 113 & 260 & 191.00 & 30.145 \\
\hline Maximum 24hr DBP & 244 & 66 & 130 & 114.44 & 10.836 \\
\hline minimum_24_SBP & 244 & 80 & 225 & 100.36 & 16.355 \\
\hline Minim_24_DBP & 244 & 60 & 127 & 64.37 & 8.519 \\
\hline
\end{tabular}

The proportion of patients with controlled BP was different as per the office and ambulatory blood pressure recordings. This proportion also differs based on the period of the ABPM studied for both sexes (Table 4).

Table 4. Proportion of controlled ABPM and OBPM by sex

\begin{tabular}{|lllll|}
\hline Parameter & Male & Female & total & Number (total=244) \\
\hline OBP controlled & 59.8 & 57.6 & 58.6 & 143 \\
\hline 24hr controlled & $42 \%$ & $47 \%$ & 45.1 & 110 \\
\hline Day time controlled & $53.6 \%$ & 58.3 & 56.1 & 137 \\
\hline Night time controlled & 19.6 & 23.5 & 21.7 & 53 \\
\hline
\end{tabular}

Correlation studies done using Pearson product-moment correlation coefficient revealed significant correlation of age, and OBP values with ABPM values.

The relationship between age and diastolic BP was investigated using Pearson product-moment correlation coefficient. Preliminary analyses were performed to ensure no violation of the assumptions of normality, linearity and homoscedasticity. There was a Small, negative correlation between the two variables, $r=-.0 .21, n=244, p<.001$, with increasing age associated with lower DBP Levels. 
An independent-samples t-test was conducted to compare the ABPM results for males and females. There was no significant difference in mean ABPM values between males (24 $\mathrm{hr} \mathrm{SBP}=$ 137(14)/82(10) and females (24 hr SBP $=136(17) / 80(11)$; t (242) $=0.46, p=.65$, two-tailed). The magnitude of the differences in the means (mean difference $=.95,95 \% \mathrm{Cl}:-3.10$ to 5.01 ) was non significant.

Comparison between patients with and without Comorbidities showed significant difference in ABPM measurements.

- Diabetics had higher 24hr systolic ABPM values compared to non diabetics (Diabetic $24 \mathrm{hr}$ SBP = $141(17) / 81$ (12) vs non diabetic (24 hr SBP $=134(15) / 80(10) ; \mathrm{t}(241)=2.8, \mathrm{p}=.005$, two-tailed). The magnitude of the differences in the means (mean difference $=6.5,95 \% \mathrm{Cl}: 1.99$ to 10.99 ) was significant. Similar results were seen for day and night SBP but not DBP.

- Dyslipidemia patients had significantly higher systolic ABPM . dyslipidemic pt $24 \mathrm{hr}$ SBP = $139(17) / 81$ (10) vs non dyslipidemics (24 hr SBP = 134(14)/80(10); t (241) = 2.2, p = .029, twotailed). The magnitude of the differences in the means (mean difference $=4.5,95 \% \mathrm{Cl}$ : 0.4 to 8.6 ) was significant. Similar result was seen for day time SBP but not night SBP or DBP.

- Patients with IHD also had poorer $24 \mathrm{hr}$ systolic ABPM control (IHD patients $24 \mathrm{hr}$ SBP = 142(18)/82(8) compared to patients without IHD (24 hr SBP = 136(16)/81(11); t (242) = 2.0, p = .046, two-tailed). The magnitude of the differences in the means (mean difference $=5.7,95 \% \mathrm{Cl}: 0.1$ to 11.4) was significant. Similar result was seen for day time SBP but not night SBP or DBP.

ABPM values were compared based on Type of anti-hypertesive drugs patient used. ARB users had poor day time SBP and DBP control. Day time ABPM in ARB users SBP $=147(23) / 87(14)$ vs patients without IHD (24 hr SBP = 134(17)/78(10); $\mathrm{t}(242)=3.0, \mathrm{p}=.003$, two-tailed). The magnitude of the differences in the means (mean difference SBP $=12.5,95 \% \mathrm{Cl}: 1.1$ to 24 and DBP Mean $=8.295 \% \mathrm{Cl}=1.2-15.2$ ) was significant. Subgroups were compared based on the Number of anti-hypertensive medications they used by the one way analysis of variance test and no difference was seen.

Patients with controlled OBP status had better ABPM values compared with those having uncontrolled OBP.

Chi square test for independence with Yates' Continuity Correction was done to compare groups with respect to control status of their ABPM measurements as per the current guidelines.

A chi-square test for independence (with Yates' Continuity Correction) indicated no significant association between gender and ABPM control status, $\chi 2(1, n=244)=.59, p=.44$, phi $=-.058$. Office BP values showed medium correlation with ABPM; the strongest positive correlation was seen between office DBP and mean night DBP , $r=-.0 .35, n=244, p<.001$, with increasing Office DBP associated with HIGHER mean night DBP Levels. 
OBPM control status had statistically significant but small degree of association of with $24 \mathrm{hr}$ ABPM control status $(x 2(1, n=244)=9.8, p=.002$, phi $=.021$. $)$, but was not correlated with night BP control status (Table 5).

Table 5. Correlation and Discrepancy between ABPM and OBP control

\begin{tabular}{|lllll|}
\hline Parameter & Controlled OBP & UnControlled OBP & $\begin{array}{l}\text { P } \\
\text { value }\end{array}$ & Phi \\
\hline Controlled 24 hr ABP & $53.8 \%$ & $\begin{array}{l}32 \%(\text { White coat } \\
\text { effect) }\end{array}$ & $=0.002$ & 0.21 \\
\hline $\begin{array}{l}\text { Uncontrolled 24 hr } \\
\text { ABP }\end{array}$ & $\begin{array}{l}46.2 \% \text { (masked uncontrolled } \\
\text { BP) }\end{array}$ & $68 \%$ & \\
\hline
\end{tabular}

Kappa measure of agreement showed Weak correlation between OBP and $24 \mathrm{HR}$ ABPM control status(kappa $=0.2$ with $p<0.001)$. OBP was able to predict controlled BP as per $24 \mathrm{HR}$ ABPM with sensitivity of $70 \%$ and specificity of $50 \%$.

Among the comorbidities, DM was inversely related with $24 \mathrm{hr}$ ABPM control status, $\chi 2(1, n=244)=10.6$, $p=0.005$, phi $=0.20$, albeit to a small degree. Other comorbidities had no significant correlation with ABPM status. The class of anti - hypertensive drug used by patients had no significant correlation with ABPM control status.

\section{Discussion}

This study is a descriptive study of ABPM conducted in Ethiopia. The results showed that only $45.1 \%$ of patients had controlled BP by ABPM compared to $58.6 \%$ based on OBP, suggesting that OBP could be over-estimating BP control in our setup. This lower rate of BP control by ABPM vs OBP in hypertensive patients on treatment has been documented in previous studies. It has been shown that the degree of OBP reduction is higher than ABPM reduction with treatment.(12) Schmieder et al noted that patients with higher pretreatment SBP levels had an even greater disproportional reduction in office SBP than in ambulatory SBP after undergoing antihypertensive treatment; in addition, these investigators noted that the white-coat effect decreased by $\approx 10 / 5 \mathrm{mmHg}$ on average after beginning antihypertensive treatment, thus contributing to the reduction of in-office BP but not in ABPM. Perhaps of equal importance, a morning recording of normal in-office BP may be at peak levels of medication while trough levels later in the day and night may be associated with hypertensive BP values seen with ABPM(13).

Significant discrepancies exist in the ability of OBP in predicting adequate ABPM control as $32 \%$ of our patients with controlled ABPM status are misclassified as having uncontrolled OBP. This scenario of white coat effect on BP control was seen in other studies. In a study of over 600 patients with uncontrolled office blood pressure (i.e., $>140 \mathrm{mmHg}$ systolic blood pressure or $>90 \mathrm{mmHg}$ diastolic blood pressure), nearly $40 \%$ of those on one or two medications and almost $30 \%$ of those on three medications had controlled blood pressure on ambulatory blood pressure monitoring (7). In a larger Spanish study of 
over 8,200 patients with resistant hypertension, $38 \%$ were attributed to white-coat effect based on ambulatory blood pressure monitoring. The true resistant hypertensive patients were younger, more frequently men, with a longer duration of hypertension and a worse cardiovascular risk profile. The group included larger proportions of smokers, diabetics, target organ damage (including left ventricular hypertrophy, impaired renal function, and micro albuminuria), and documented cardiovascular disease (8). Muxfeldt et al., in a cohort of 286 hypertensive subjects with uncontrolled BP, found that $43.7 \%$ had white coat $\mathrm{RH}$, (office BP $>140 / 90 \mathrm{mmHg}$ and daytime $\mathrm{BP}<135 / 85 \mathrm{mmHg}$ ) and less target organ damage compared to the true resistant hypertensives (14).

This similar finding from the above studies in different populations confirm the limitations of OBP in assessing adequacy of BP control, which can ultimately lead to unnecessary treatment escalation with possible increase in side effects.

On the other end of the spectrum, patients with apparently controlled OBP can have unmasking of their poor BP control during ABPM. In our study, $46.2 \%$ of individuals with controlled OBP were shown to have masked BP elevation (masked uncontrolled hypertension) with 24 hour ABPM. In support, Pierdomenico et al. in a cohort of 742 treated hypertensive subjects, 426 apparently responders and 276 apparently resistant, found that 126 subjects (29.5\% of the apparently responders) had masked hypertension and 146 (52.8\% of the apparent resistant) had white coat RH. In the same study, in the follow-up period cardiovascular risk was higher in masked hypertensives (masked versus responder hypertensives, relative risk (RR) 2.28, 95\% confidence interval (Cl) 1.1-4.7, ) and in true resistant hypertensives (true resistant versus responder hypertensives, $\mathrm{RR} 2.94,95 \% \mathrm{Cl} 1.02-8.41$, ) (9).

A similar study from Egypt revealed $33.2 \%$ of apparent responders by OBP had masked uncontrolled hypertension (MUCH) according to 24-h ABPM criteria (15). This study also showed the dominant contribution of nocturnal hypertension for $\mathrm{MUCH}$, which was solely attributable to an elevated nocturnal BP almost double that due to daytime BP elevation ( $57.3 \%$ vs. $27.1 \%, \mathrm{P}<0.001)$. Our study also showed the dominant contribution of nocturnal hypertension for $\mathrm{MUCH}$ compared to day time BP elevation(75\% vs $36 \%)$.

Our study shows that half of our study population had uncontrolled BP by both OBP and ABPM criteria. Similarly, only $40 \%$ of patients had controlled OBP in a recent Ethiopian study (6). Suboptimal BP control has been reported in other many studies worldwide; in India, uncontrolled BP was found to be $62.3 \%$; and in the USA, $51.2 \%$ of the participants were found to have uncontrolled $\mathrm{BP}(16)$.

These data show the poor efficiency of current HTN management throughout the range of healthcare facilities at different levels of development.

There was no difference in ABP control status between the sexes. Increasing age was associated with lower DBP values as would be expected from the vascular changes related to ageing starting from the $6^{\text {th }}$ decade. This changes in BP was first shown in the Framingham heart study. 
In our study, Diabetic patients were shown to have significantly higher 24 hour and night time ABPM values and lower rate of ABPM control status compared to non diabetics. This has been shown in ABPM studies done on diabetics.WCH seems to be less frequent, and masked HTN is more frequent in diabetic patients and seems to be associated with increased organ damage(17).

Dyslipidemic patients and those with IHD also had significantly higher mean 24 hour and day time SBP values. Similar trends have been reported in other studies done using ABPM.

In an Italian study it was shown that the true resistant hypertensive patients had a worse cardiovascular risk profile. The group included larger proportions of smokers, diabetics, target organ damage (including left ventricular hypertrophy, impaired renal function, and micro albuminuria), and documented cardiovascular disease (8).

Among the treatment agents, ARB users had higher mean ABPM value. This is likely related to very small number of patients using ARB affecting the statistical analysis. The number of medications used did not result in significant difference of BP control in our study. Number of antihypertensive medications was not significant determinant of treatment efficacy in our study. Similar findings were noted by Pierdomenico et al. where percentage of subjects receiving single, double, and multiple therapy was not different between responder and masked hypertension $(58.2 \%$ v $62.7 \%, 28.2 \% \vee 26.2 \%$, and $13.7 \% \mathrm{v}$ $11.1 \%$, respectively, $\mathrm{P}=$ not significant [NS]). Drug classes were not different. Use of three or more drugs

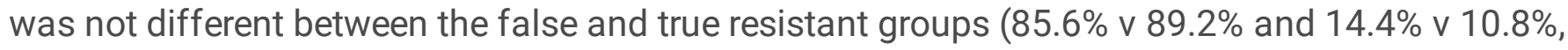
respectively, PNS), and drug types were not different (about $90 \%$ in each group received a diuretic)(9).

\section{Conclusion}

This study shows the high burden of uncontrolled BP in this cohort. It also shows the significant advantages of ABPM in assessing adequacy of BP control with additional BP parametrs of prognostic importance and complementary to OBP can help clinicians for more informed decision making. The increased prevalence of uncontrolled BP status in patients with comorbidities like diabetes shows the need to increase the use of ABPM in this high risk population. Further studies to incorporate more patient cohorts with prospective models can further elucidate the role of ABPM in our setup.

\section{Declarations}

- Ethics approval and consent to participate - The Ethics Committees of Addis Ababa University, Tikur Anbesa Specialized Hospital, Ethiopia, approved the study protocol and consent form (Dr. Adamu Adissie, Addis Ababa University Institutional Review Board chairperson). The participants gave informed written consent to the study. The study was conducted in accordance with the standard international and national scientific and ethical guidelines.

- Consent for publication - obtained from all patients via informed written consent. 
- Availability of data and materials - The datasets used and/or analysed during the current study are available from the corresponding author on reasonable request

- Competing interests - None

- Funding - Addis Ababa University

- Authors' contributions - "Mulualem Alemayehu analyzed and interpreted the patient data regarding the ambulatory blood pressure control pattern. All authors read and approved the final manuscript."

- Acknowledgements - none

- Authors' information (optional) -

\section{References}

1. WHO. Noncommunicable Diseases Country Profiles 2018. World Heal Organ. 2018;223.

2. Ethiopian Public Health Institute, Federal Ministry $f$ Health and WHO. ETHIOPIA STEPS REPORT ON RISK FACTORS FOR NON-COMMUNICABLE DISEAES AND PREVALENCE OF SELECTED NCDS ETHIOPIA STEPS REPORT ON RISK FACTORS FOR CHRONIC NON- COMMUNICABLE DISEASES AND. 2016;(December):1-13.

3. Williams B, Masera G. 2018 ESC / ESH Guidelines for the management of arterial hypertension The Task Force for the management of arterial hypertension of the. 2018.

4. Unger T, Borghi C, Charchar F, Khan NA, Poulter NR, Prabhakaran D, et al. 2020 International Society of Hypertension Global Hypertension Practice Guidelines. Hypertension. 2020;75(6):1334-57.

5. Kayima J, Wanyenze RK, Katamba A, Leontsini E, Nuwaha F. Hypertension awareness, treatment and control in Africa: A systematic review. BMC Cardiovasc Disord. 2013;13.

6. Berhe DF, Taxis K, Haaijer-Ruskamp FM, Mulugeta A, Mengistu YT, Mol PGM. Hypertension treatment practices and its determinants among ambulatory patients: Retrospective cohort study in Ethiopia. BMJ Open. 2017;7(8):1-11.

7. Brown MA, Buddle ML, Martin A. Is Resistant Hypertension Really Resistant? 2001;7061(01):1263-9.

8. Sierra A De, Banegas R, Gorostidi M, Cruz JJ De, Armario P, Oliveras A, et al. Clinical Features of 8295 Patients With Resistant Hypertension Classified on the Basis of Ambulatory Blood Pressure Monitoring. 2011;898-902.

9. Pierdomenico SD, Lapenna D, Bucci A, Di Tommaso R, Di Mascio R, Manente BM, et al. Cardiovascular outcome in treated hypertensive patients with responder, masked, false resistant, and true resistant hypertension. Am J Hypertens. 2005;18(11):1422-8.

10. O'Brien E, White WB, Parati G, Dolan E. Ambulatory blood pressure monitoring in the 21 st century. J Clin Hypertens. 2018;20(7):1108-11.

11. Banegas R, Sobrino J, Rodrı F, Sierra A De, Cruz JJ De, Gorostidi M, et al. Hypertension Treatment and Control Effectiveness of Blood Pressure Control Outside the Medical Setting. 2006;62-8. 
12. Franklin SS, O'Brien E, Thijs L, Asayama K, Staessen JA. Masked hypertension a phenomenon of measurement. Hypertension. 2015;65(1):16-20.

13. Schmieder RE, Schmidt ST, Riemer T, Dechend R, Hagedorn I, Senges J, et al. Disproportional decrease in office blood pressure compared with 24-hour ambulatory blood pressure with antihypertensive treatment: Dependency on pretreatment blood pressure levels. Hypertension. 2014;64(5):1067-72.

14. Muxfeldt ES, Salles GF. How to use ambulatory blood pressure monitoring in resistant hypertension. Hypertens Res. 2013;36(5):385-9.

15. Youssef G, Nagy S, El-gengehe A, Abdel Aal A, Hamid MA. Masked uncontrolled hypertension: Prevalence and predictors. Egypt Hear J [Internet]. 2018;70(4):369-73. Available from: https://doi.org/10.1016/j.ehj.2018.10.001

16. McDonald M, Hertz RP, Unger AN, Lustik MB. Prevalence, awareness, and management of hypertension, dyslipidemia, and diabetes among United States adults aged 65 and older. Journals Gerontol - Ser A Biol Sci Med Sci. 2009;64(2):256-63.

17. Pierdomenico SD, Cuccurullo F. Ambulatory blood pressure monitoring in type 2 diabetes and metabolic syndrome: A review. Blood Press Monit. 2010;15(1):1-7. 